Ann. Zootech., I979, 28 (I), Io9-II9.

\title{
Essai de détermination du besoin en phosphore des pintades reproductrices
}

\author{
B. SAUVEUR
}

avec la collaboration technique de Maryse GarkeaU et J. P. BRILLARD

\author{
Station de Recherches Avicoles, \\ Centre de Recherches de Tours, I.N.R.A., \\ Nouzilly, 37380 Monnaie, France
}

\begin{abstract}
Résumé
2 ro pintades reproductrices issues de 2 souches commerciales différentes ont été logées en cages individuelles à partir de 25 semaines d'âge et étudiées pendant 9 périodes de production de 28 jours avec insémination artificielle hebdomadaire. 6 taux de phosphore alimentaire compris entre 2,5 et $5,0 \mathrm{~g} / \mathrm{kg}$ de $\mathrm{P}$ disponible $(4,8$ à $7,3 \mathrm{~g} / \mathrm{kg}$ de $\mathrm{P}$ total) ont été comparés en présence d'un taux unique de calcium $(32 \mathrm{~g} / \mathrm{kg}$ ). Aucun effet significatif n'est visible sur la mortalité, le nombre d'œufs pondus, le poids moyen de l'œuf, la consommation journalière d'aliment et l'indice de consommation. A l'opposé, 4 incubations effectuées entre la $4^{\mathrm{e}}$ et la $9^{\mathrm{e}}$ période avec un total d'environ 500 œufs /régime montrent que l'éclosivité des œufs est abaissée si le régime contient moins de 4,o g/ $\mathrm{kg}$ de $\mathrm{P}$ assimilable. Le besoin en phosphore de la pintade reproductrice parait donc se situer comme celui de la dinde au voisinage de $6,5 \mathrm{~g}$ de $\mathrm{P}$ total $/ \mathrm{kg}$ d'aliment $(4 \grave{a} 4,5 \mathrm{~g} / \mathrm{kg}$ de $\mathrm{P}$ disponible).
\end{abstract}

\section{Introduction}

Il a été montré durant les six dernières années que le besoin alimentaire en phosphore des poules pondeuses est inférieur aux valeurs précédemment ađmises. Ainsi, au lieu des 7 à $8 \mathrm{~g}$ de phosphore total $/ \mathrm{kg}$ d'aliment classiquement prescrits (soit environ 5 à $5,5 \mathrm{~g} / \mathrm{kg}$ de $\mathrm{P}$ assimilable (I); FRITZ, I974), on rencontre fréquemment maintenant des recommandations comprises entre 4,5 et $5,5 \mathrm{~g} / \mathrm{kg}$ de $\mathrm{P}$ total. Ceci est vrai aussi bien pour des poules de type Leghorn productrices d'œufs blancs (Scott, Antilion et Muldienhoff, I975; Owings, Seil et Balioun, I977) que pour celles de type Rhode Island productrices d'œufs colorés ('ToNDEREAU, I972).

(1) Phosphore assimilable calculé selon la méthode habituelle pour les volailles en estimant que le phosphore d'origine végétale n'est disponible qu'à 30 p. I00. 
Les résultats sont plus nuancés pour les animaux reproducteurs. Chez la poule reproductrice, WALDROuP et al. (I967) estiment à $2 \mathrm{~g} / \mathrm{kg}$ la quantité de phosphore minéral à ajouter au régime, ce qui doit conduire à un apport de $\mathrm{P}$ total encore voisin de $5,5 \mathrm{~g} / \mathrm{kg}$; MILLER (I976) rapporte également que l'éclosivité des œufs est réduite lorsque le taux de $\mathrm{P}$ total du régime est inférieur à $4 \mathrm{~g} / \mathrm{kg}$. Chez la dinde reproductrice, SEWELI, et al. (I972) trouvent de même qu'une supplémentation en phosphore minéral est indispensable pour obtenir une éclosivité maximale des œufs; selon PotTrer, Leighton et CHU (I974) et WALDroup, MAXEy et LUTHER (I974) cette supplémentation doit être voisine de $3 \mathrm{~g} / \mathrm{kg}$ ( $\mathrm{P}$ total voisin de $6,5 \mathrm{~g} / \mathrm{kg}$ ) tandis que MILLER, BRADLEY et FERGUSON (I976) ne trouvent pas de différence significative entre des supplémentations de I et $4 \mathrm{~g} / \mathrm{kg}$. Selon ATKINsor et al. (I976), l'éclosivité des œufs fertiles augmente encore lorsque le phosphore disponible du régime est accru de 4,5 à $6,5 \mathrm{~g} / \mathrm{kg}$ mais la fertilité des dindes ellemême est abaissée.

Aucune donnée similaire ne permet de connaître l'effet du phosphore du régime sur les performances de la pintade reproductrice malgré l'importance de cette espèce dans la production avicole française. Nous avons donc cherché à combler en partie cette lacune en ne retenant, dans un but de simplification, qu'un seul taux calcique $(32 \mathrm{~g} / \mathrm{kg})$. Six niveaux d'apport phosphoré compris entre 4,8 et 7,3 $\mathrm{g} / \mathrm{kg}$ de $\mathrm{P}$ total ont ainsi été étudiés.

\section{Matériel et méthodes}

\section{Animaux}

2 Io femelles provenant à égalité de deux souches commerciales $\mathrm{X}$ et $\mathrm{Y}$ on $\mathrm{t}$ été réparties en 6 lots de 35 correspondant aux 6 régimes alimentaires. Chaque lot a été subdivisé en 5 répétitions de 7 animaux juxtaposés, ces répétitions étant réparties au hasard entre les 2 façades et les 3 étages d'une même batterie. Suivant les régimes, il a donc été réalisé, pour chaque souche, 2 ou 3 répétitions d'un même régime.

I,es animaux âgés de 25 semaines ont été placés en cages individuelles le 20 août I975, soit environ 2 semaines avant l'apparition des premiers œufs. La durée d'éclairement du local a été progressivement augmentée de 9 à $\mathrm{I}_{4} \mathrm{~h} / \mathrm{jour}$ entre le 25 aôtt et le 29 septembre, à raison d'une heure d'accroissement par semaine.

\section{Régimes}

La composition et les caractéristiques calculées du régime de base sont données dans le tableau I. Les 6 régimes utilisés ne diffèrent que par leur teneur en phosphore croissant de 4,8 à $7,3 \mathrm{~g} / \mathrm{kg}$ de $\mathrm{P}$ total (tabl. 2). En estimant le phosphore végétal disponible à $30 \mathrm{p}$. IOo, ceci équivaut à des taux de $\mathrm{P}$ disponible compris entre 2,5 et $5,0 \mathrm{~g} / \mathrm{kg}$ avec une progression de $0,5 \mathrm{~g} / \mathrm{kg}$.

Ces régimes (toujours présentés en farine) ont été distribués ad libitum à partir de l'entrée en ponte; durant les semaines précédant celles-ci, une transition progressive a été effectuée en mélangeant ces aliments avec ceux préalablement distribués aux animaux. 


\section{TABLEAU I}

Composition du régime de base ( $p$. Ioo)

Ingredients of basal diet

Maïs broyé. . . . . . . . . . . 60

(Ground maize)

Huile végétale . . . . . . . . . . ${ }_{2}$

(Vegetable oil)

Graisse animale

(Animal fat)

Tourteau de soja 50 . . . . . . . . 24

(Soyabean meal)

Farine de luzerne . . . . . . . . . 2

(Iucerne)
Chlorure de sodium . . . . . . . 0,35

(Sodium chloride)

Oligo-éléments minéraux . . . . 0,20

(Trace minerals)

Mélange vitaminique . . . . . . 0,50

(Vitamin mixture)

DL méthionine . . . . . . . . 0,03

Mélange minéral (cf. tabl. 2). . . . 8,92 (Mineral mixture)

$\overline{10,00}$

\section{Caractéristiques calculées}

Calculated composition

Ínergie métabolisable $(\mathrm{kJ} / \mathrm{kg})$. . . . . . . . . . . . . . . . . . . . . . . . I 2460

(Metabolisable enevgy)

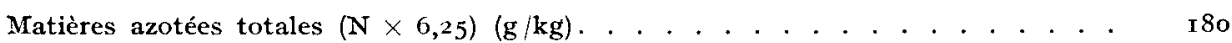

(Crude protein)

Lysine $(\mathrm{g} / \mathrm{kg})$. . . . . . . . . . . . . . . . . . . . . . . . . . . . . . . 9

Acides aminés soufrés totaux $(\mathrm{g} / \mathrm{kg})$. . . . . . . . . . . . . . . . . . . . . 6,9

(Methionine + cystine)

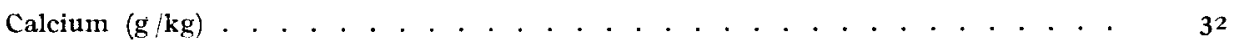

\section{TABLEAU 2}

Teneur en phosphore des régimes ( $g / k g$ )

Phosphorus contents of the diets

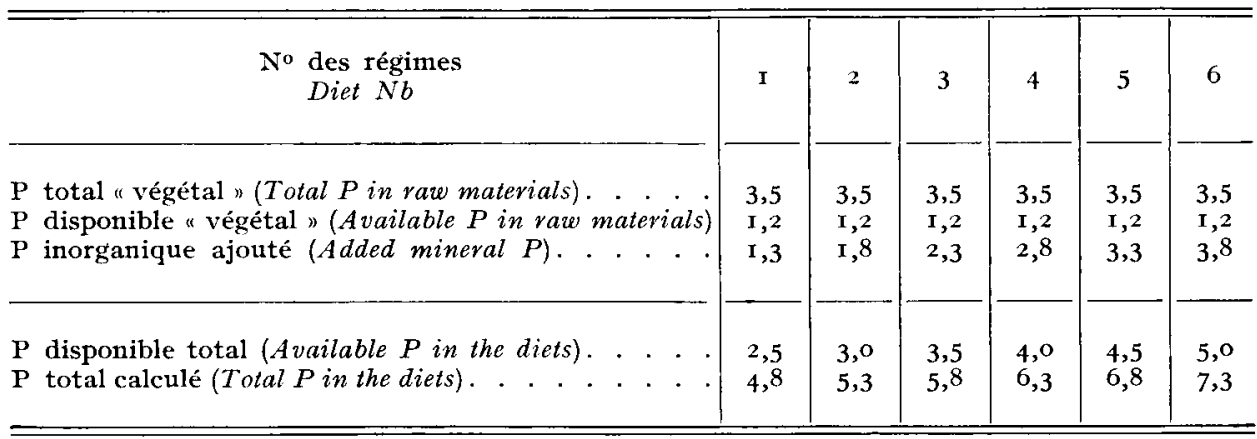




\section{Mesures}

La ponte individuelle a été en registrée quotidiennement pendant 9 périodes de 28 jours. Le poids moyen de l'œuf a été estimé par pesée de 3 œufs consécutifs par poule au cours des $4^{\mathrm{e}}, 5^{\mathrm{e}}, 7^{\mathrm{e}}, 8^{\mathrm{e}}$ et $9^{\mathrm{e}}$ période. La consommation d'aliment a été déterminée en fin de chaque période pour chaque répétition : on dispose donc de 2 ou 3 valeurs pour chaque régime et chaque souche.

Quatre tests d'éclosivité ont été réalisés entre la $4^{\mathrm{e}}$ et la $9^{\mathrm{e}}$ période, après insémination artificielle hebdomadaire. L'ensemble de ces tests représente entre 440 et 575 œufs incubés par régime, soit un total d'environ 3000 cufs.

\section{Calculs}

Le nombre d'œufs pondus par poule et les intensités de ponte ont été calculés selon 2 critères différents :

- par rapport aux poules " départ ": poules étant effectivement entrées en ponte (ayant pondu au moins 5 œufs). Ce critère a conduit à l'élimination de 5 animaux ( $I$ en $R_{3} X, 2$ en $R_{1} Y$, et 2 en $R_{4} Y$ );

- par rapport aux poules "présentes " (" journées pondeuses ") : mêmes animaux mais en ne prenant en compte que les jours de présence effective avant décès.

Au cours des calculs, après analyses des variances suivant des plans factoriels, les comparaisons multiples de moyennes ont été réalisées par un test de NewmanKeuls (DagneI,IE, I975).

\section{Résultats}

\section{Mortalité}

La mortalité cumulée (moyenne par souche) est représentée sur la figure I en dessous des courbes d'intensité de ponte; elle apparaît anormalement élevée, surtout en fin de période de ponte. L,es valeurs totales sont de I9, I p. roo pour la souche $\mathrm{X}$ et $39,3 \mathrm{p}$. Ioo pour la souche $\mathrm{Y}\left(x^{2}\right.$ de comparaison entre souches $=5.54 ; \mathrm{P}<0,025)$. En revanche, les différents régimes n'ont pas d'effet sur le pourcentage de mortalité.

\section{Nombre d'aufs pondus}

Les nombres d'œufs pondus en 9 périodes ainsi que les intensités de ponte correspondantes sont rapportés dans le tableau 3. La figure I représente l'évolution temporelle de l'intensité de ponte calculée sur la base des poules "présentes " (" journées pondeuses "). Il apparaît clairement que, quel que soit le critère de production considéré, la souche $\mathrm{X}$ a une ponte supérieure à la souche $\mathrm{Y}$; la différence est de 5,7 p. Ioo en moyenne par rapport aux poules "présentes" $(\mathrm{P}<0,0 \mathrm{I})$ et $8 \mathrm{p}$. Ioo par rapport aux poules " départ " $(\mathrm{P}<0,00 \mathrm{I})$, cette deuxième expression incluant la plus forte mortalité de la souche $\mathrm{Y}$ déjà signalée. 


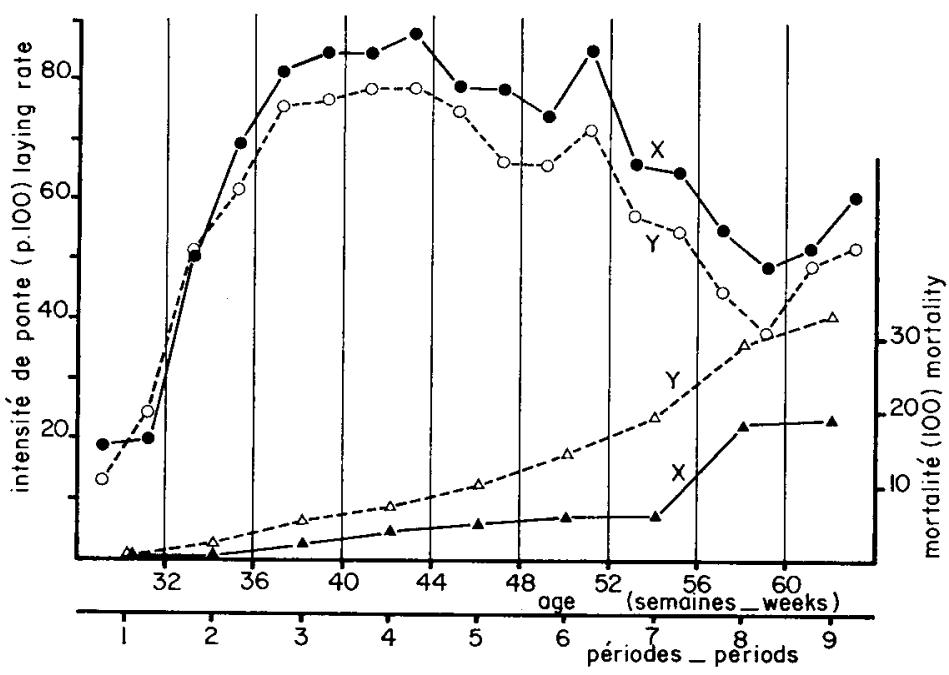

HIG. I. - Évolution des moyennes d'intensité de ponte et de mortalité dans les deux souches de pintades $X$ et $Y$. Les taux de phosphore alimentaire étudiés n'infuent pas sur ces paramètres. (Evolution of laying vate and mortality in the two strains $X$ and $Y$ of guinea-fowl breeders. These parameters were not significantly affected by the studied dietary phosphorus levels).

Pour ce qui concerne l'effet des régimes, il pourrait sembler à première vue que le taux le plus élevé de phosphore soit favorable à la souche $\mathrm{X}$. Cependant une analyse de la variance effectuée selon un plan factoriel indique clairement que à l'intérieur de chacun des 2 modes d'expression utilisés (poule " départ " ou poule "présente ") les moyennes des 6 régimes ne diffèrent pas significativement et qu'il n'existe pas d'interaction significative "souche $x$ niveau de phosphore". Il semble donc, en première approximation, qu'un taux de phosphore disponible aussi bas que $2,5 \mathrm{~g} / \mathrm{kg}$ assure une production d'œufs normale.

\section{Poids de l'ouf}

Les résultats des 5 sondages effectués sur le poids de l'œuf sont rapportés dans le tableau 4 . I1 y apparaît encore un très net effet souche, la souche Y présentant suivant les périodes une supériorité de 2,5 à $4,5 \mathrm{~g} /$ /uf par rapport à la souche $\mathrm{X}$.

Une analyse de variance "intra-périodes " ne montre aucun effet significatif du taux de phosphore. Il ne se manifeste de façon significative que sous forme d'interaction avec l'effet souche lors des $5^{\mathrm{e}}$ et $8^{\mathrm{e}}$ périodes, interaction qui paraît due surtout à un classement très différent des régimes 2 et 3 dans les 2 souches sans qu'aucune justification ne puisse être donnée à cette différence.

Sur l'ensemble des 5 périodes étudiées, une analyse de variance "intra-souches » fait apparaître chez la souche $X$ une réduction légère mais significative du poids de l'œuf avec les deux taux de phosphore les plus bas $(4,8$ et $5,3 \mathrm{~g} / \mathrm{kg}$ de $\mathrm{P}$ total); cependant, le régime $3(5,8 \mathrm{~g} / \mathrm{kg}$ de $\mathrm{P}$ total) donne un poids d'œuf aussi élevé que les régimes 5 et 6 . Chez la souche $Y$, les variations entre régimes sont du même ordre de grandeur mais inversées, les régimes I et 2 conduisant aux poids d'œufs les plus élevés. A noter qu'aucune des 2 souches ne présente d'interaction significative entre le taux de phosphore et l'effet "périodes". 


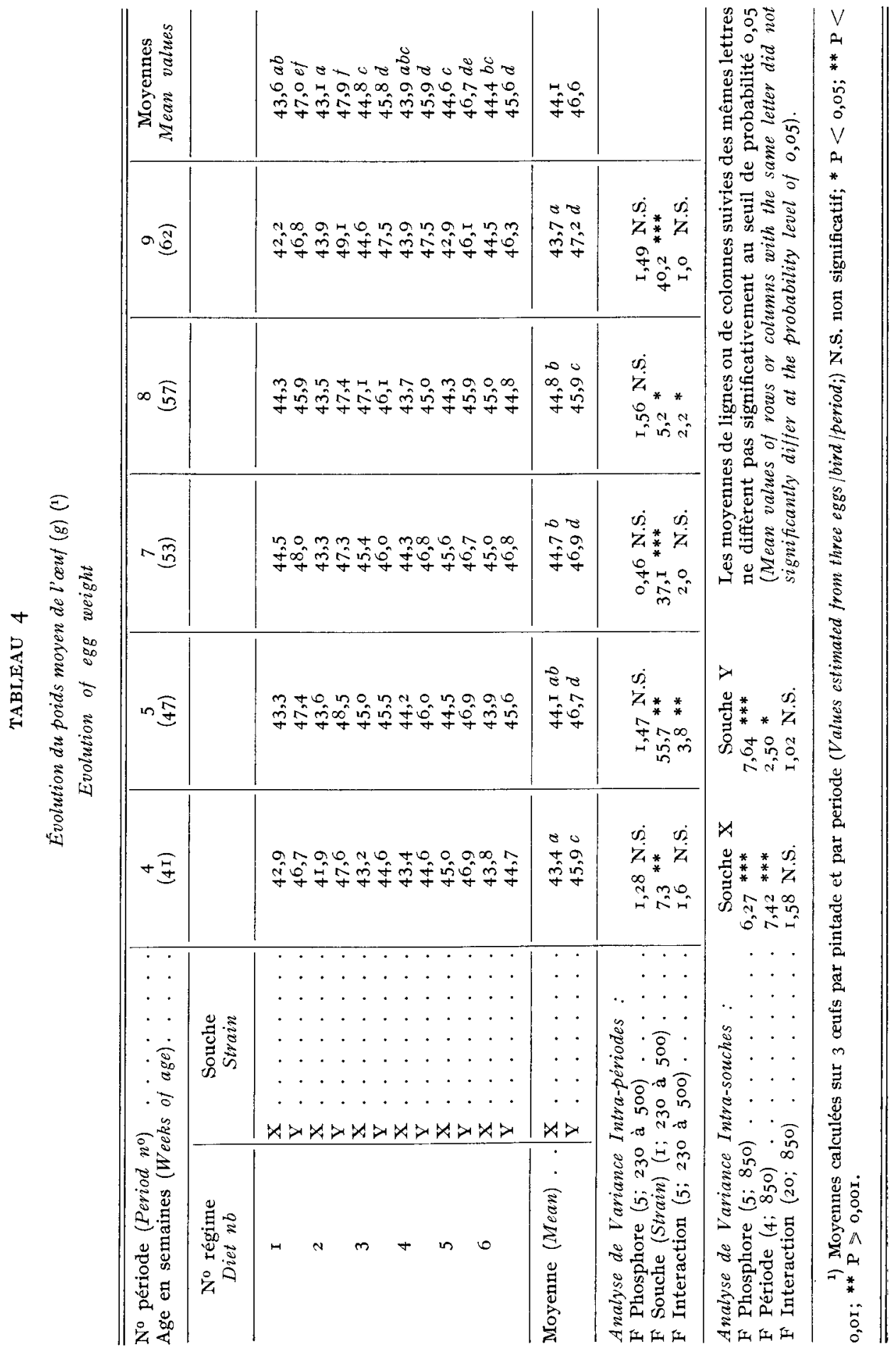




\section{Consommation d'aliment}

L'estimation pratiquée pour la consommation journalière d'aliment inclut vraisemblablement une part non négligeable de gaspillage. Quoi qu'il en soit, cette consommation ne varie significativement ni en fonction du taux de phosphore du régime, ni avec la souche. Elle varie par contre en fonction du temps : égale à II $2 \mathrm{~g} / \mathrm{j} /$ pintade au moment de l'entrée en ponte, elle atteint son maximum (I53 g) lors du pic de ponte ( $3^{\mathrm{e}}$ période) et régresse ensuite jusqu'à roo $\mathrm{g} / \mathrm{j} /$ pintade pendant la $9^{\mathrm{e}}$ période.

\section{Indice de consommation}

Compte tenu du faible intérêt du critère "poids d'œuf exporté " dans le cas d'animaux reproducteurs, on a calculé un indice de consommation ne tenant compte que du nombre d'œufs produits. L'évolution de cet indice entre la $3^{\mathrm{e}}$ et la $9^{\mathrm{e}}$ période de ponte est représentée sur la figure 2 (moyenne par souche seulement). L'analyse de variance de ce paramètre n'indique en effet aucune différence significative entre régimes $(\mathrm{F}=0,9)$ bien que le régime 6 paraisse donner un résultat meilleur que la moyenne des autres (I54 contre I65 g d'aliment/ouf).

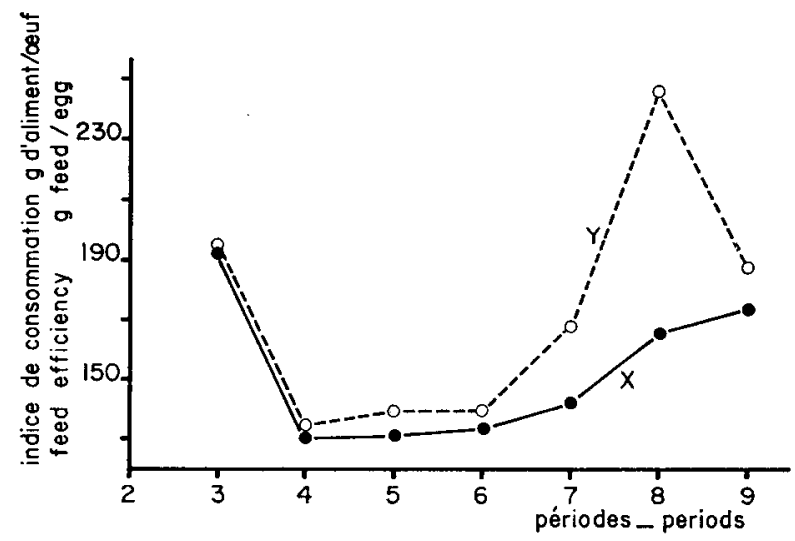

FIG. 2. - Évolution de l'indice de consommation ( $g$ d'aliment consommé par ceuf produit) dans les deux sonches de pintades $X$ et $Y$. Les taux de phosphore alimentaire étudiés ne modifient pas significativement ce paramètre.

(Evolution of feed efficiency-expressed as $g$ of feed per egg-in the two strains $X$ and $Y$ of guineafowl breeders. There was no significant effect of the studied phosphorus levels on this criterian).

Il existe en revanche des effets hautement significatifs :

- du stade de production $(\mathrm{F}=26 ; \mathrm{P}<0, \mathrm{OOI}):$ 'indice est minimum après le pic de ponte au voisinage de $\mathrm{I} 35 \mathrm{~g} /$ euf puis augmente en fin de production;

- de la souche de pintade $(\mathrm{F}=20 ; \mathrm{P}<0,00 \mathrm{I})$ : les valeurs moyennes sont respectivement de $\mathrm{I}_{53}$ et $\mathrm{I} 73 \mathrm{~g}$ d'aliment/ouf pour les souches $\mathrm{X}$ et $\mathrm{Y}$.

A ces effets simples s'ajoutent des interactions significatives entre stade de production et souche puisqu'il est bien visible sur la figure 2 que la dégradation des performances de la souche $\mathrm{Y}$ n'intervient essentiellement que pendant la $8 \mathrm{e}$ période. 


\section{Résultats d'incubation}

Les résultats progressivement cumulés des 4 incubations effectuées sont donnés par la figure 3 . Compte tenu du faible nombre d'œufs incubés (environ $500 /$ régime) il n'est pas possible de dissocier dans les calculs les 2 souches $\mathrm{X}$ et $\mathrm{Y}$.

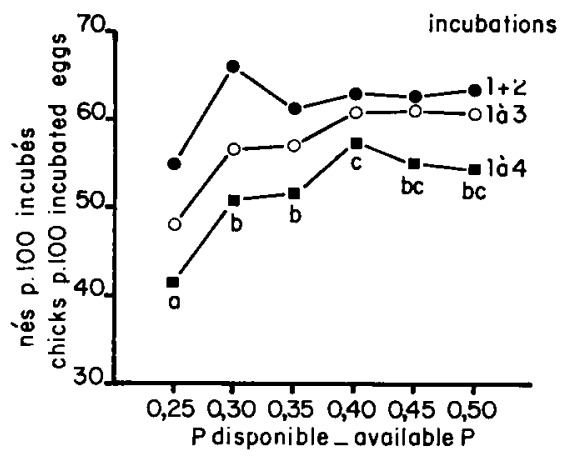

FIG. 3. - Ellets du taux de phosphore disponible du régime sur l'éclosivité des outs de pintade. Courbes cumulées de quatre incubations. Sur la courbe inférieure, les points suivis des mêmes lettres ne diffèrent pas significativement au seuil de probabilité 0,05 .

(Effects of the level of available phosphorus in the diet of guinea-fowl breeders on egg hatchability expressed as chicks p. roo incubated eggs. On inferior curve cumulating four incubations, points followed by the same letter were not significantly different at the 0.05 probability level).

Sur l'ensemble des incubations (courbe inférieure), le régime I (contenant $2,5 \mathrm{~g} / \mathrm{kg}$ de $\mathrm{P}$ disponible) conduit à un retard significatif d'éclosivité d'au moins ro p. Ioo par rapport aux 5 autres régimes. A l'intérieur de ceux-ci les $n^{\circ} 2$ et 3 paraissent également inférieurs aux trois suivants mais ne diffèrent significativement que du régime $4 ; 3$ incubations sur 4 reflètent cette tendance générale. Il semble donc que le besoin en phosphore des pintades, estimé par l'éclosivité des œufs, soit supérieur à celui déduit des simples performances de ponte et qu'il doive être estimé au moins égal à $4 \mathrm{~g} / \mathrm{kg}$ de $\mathrm{P}$ disponible $(6,3 \mathrm{~g} / \mathrm{kg}$ de $\mathrm{P}$ total).

La dégradation progressive de l'éclosivité au cours du temps peut également être soulignée puisqu'elle évolue en moyenne de 64 à 36 p. Ioo des oufs incubés entre la $4^{\mathrm{e}}$ et la $9^{\mathrm{e}}$ période de ponte.

\section{Discussion et conclusion}

Le but du présent essai était d'étudier l'effet du taux de phosphore du régime sur les performances de pintades reproductrices; 6 taux d'apport phosphoré compris entre 4,8 et $7,3 \mathrm{~g} / \mathrm{kg}$ de $\mathrm{P}$ total (soit 2,5 à $5,0 \mathrm{~g} / \mathrm{kg}$ de $\mathrm{P}$ assimilable) ont donc été comparés.

Il doit d'abord être souligné que la 8 e période d'étude s'est avérée particulièrement mauvaise quel que soit le critère considéré : on y a noté en effet une forte mortalité, une chute de ponte importante, une stagnation du poids de l'œuf, une forte dégradation de l'indice de consommation et une baisse d'éclosivité. Ce phéno- 
mène inexpliqué est d'autant plus surprenant qu'il n'a pas eu son correspondant dans une pièce voisine du même bâtiment abritant des animaux de même âge et de même origine.

Malgré cet incident les présents résultats permettent d'avoir pour la première fois une estimation du besoin en phosphore des pintades reproductrices en présence d'un taux de calcium de $32 \mathrm{~g} / \mathrm{kg}$.

Si l'on se fie uniquement au paramètre de production d'œufs, il semble qu'un taux de $2,5 \mathrm{~g} / \mathrm{kg}$ de phosphore disponible $(4,8 \mathrm{~g} / \mathrm{kg}$ de $\mathrm{P}$ total) serait suffisant pour les pintades reproductrices; une légère nuance doit être apportée dans le cas d'une des 2 souches étudiées dont le poids de l'œuf paraît légèrement réduit en dessous de $3,5 \mathrm{~g} / \mathrm{kg}$ de $\mathrm{P}$ disponible. Les résultats d'incubation, quant à eux, conduisent à rejeter le plus faible niveau de phosphore $(2,5 \mathrm{~g} / \mathrm{kg}$ de $\mathrm{Pd})$ et tendent à faire préférer une teneur minimale de $4,0 \mathrm{~g} / \mathrm{kg}$ de $\mathrm{P}$ disponible dans la ration $(6,3 \mathrm{~g} / \mathrm{kg}$ de $\mathrm{P}$ total).

Bien que ce résultat doive être considéré avec prudence du fait du nombre assez faible d'œufs incubés, il va tout à fait dans le sens de conclusions obtenues chez des reproductrices d'autres espèces et en particulier chez la dinde. Comme il a été rappelé en introduction, l'éclosivité des œufs de dinde s'accroît avec la quantité de phosphore minéral distribué aux reproductrices et ceci jusqu'au niveau de $3 \mathrm{~g} / \mathrm{kg}$ (PoTTER et al., I 974; WALDRoup et al., I974). Ce seuil correspond très sensiblement au régime $n^{\circ} 4$ du présent essai en dessous duquel l'éclosivité diminue; ceci tend à démontrer que le besoin en phosphore de la pintade reproductrice doit être voisin de celui de la dinde et proche de $6,5 \mathrm{~g} / \mathrm{kg}$ de phosphore total (4 à $4,5 \mathrm{~g} / \mathrm{kg}$ de $\mathrm{P}$ disponible) lorsque l'apport calcique est de $32 \mathrm{~g} / \mathrm{kg}$.

Accepté pour publication en novembre 1978 .

\section{Remerciements}

Le présent travail a été réalisé avec la participation contractuelle des Sociétés S.A. BEGHIN Frères, S.A. Galior, Lesieur-Sodeva et de la Centrale Coopérative des Productions Animales.

\section{Summary}

\section{Effects of 6 levels of dietary phosphorus on performance of guinea-fowl breeders.}

A total of 210 guinea-fowl breeders issued from two different commercial strains were individually caged at 25 weeks of age and then kept in production during nine 28 day-periods with weekly artificial insemination. Six dietary phosphorus levels from 2.5 to $5.0 \mathrm{~g} / \mathrm{kg}$ of available phosphorus (4.8 to $7.3 \mathrm{~g} / \mathrm{kg}$ of total phosphorus) were compared in the presence of the same dietary calcium level $(32 \mathrm{~g} / \mathrm{kg})$. Mortality, egg-production, egg weight, daily feed intake and feed conversion ratio were not significantly affected by dietary phosphorus. On the other hand four incubations made between the ${ }_{4}$ th and the 9 th laying period with a total of about 500 eggs / diet showed a decrease in hatchability when the available phosphorus level of the diet was under $4.0 \mathrm{~g} / \mathrm{kg}$. Thus, the phosphorus requirement of guinea-fowl breeders seems to be close to 6.5 $\mathrm{g} / \mathrm{kg}$ of total phosphorus $(4.0-4.5 \mathrm{~g} / \mathrm{kg}$ of available $\mathrm{P})$, a value close to that recommended for turkey breeders. 


\section{Références bibliographiques}

Atkinson R. L., Bradley J. W., Couch J. R., Ferguson T. M., Krueger W. F., 1976. Relationship of supplemental biotin, phosphorus level and calcium level to reproductive performance of turkeys. Nutr. Rep. Int., 13, 237-246.

DAGNelie P., 1975. Théorie et méthodes statistiques. Vol. 2 : Les méthodes de l'inférence statistique. Presses agronomiques de Gembloux.

Frirz J. C., 1974. Phosphorus requirements of poultry. Proc. Maryland Nutr. Conf., 96-I02.

Militer D. H., BRAdLEY J. W., Ferguson T. M., I976. Reproductive performance of broad breasted white turkeys in relation to dietary phosphorus. Poultry Sci., 55, 248I-2483.

MII,ER E. R., r976. Calcium and phosphorous effects on hatchability. Poultry Digest, $\mathrm{n}^{\circ}$ de mars 1976, I 29-130 (Abstr.).

Owings W. J., SEll J. L., Balloun S. L., I977. Dietary phosphorus needs of laying hens. Poultry Sci., 56, 2056-2060.

Potter L. M., Leighton A. T., Chu A. B., I974. Calcium phosphorus and Nopgro as variables in diets of breeder turkeys. Poultry Sci., 53, I5-22.

Scotr M. L., ANTILlon A., MUllenhofF P. A., I975. The effects of levels of calcium, phosphorus and vitamin $\mathrm{D}$ on bone development and eggshell quality in modern laying hens. Proc. Cornell Nutr. Conf., 77-80.

SEWELI, C. E., ATKinson R. L., Couch J. R., FERGuson T. M., I972. The effect of supplemental Phosphorus on the reproductive performance of turkey hens and the subsequent effect upon the poults. Poultry Sci., 51, 792-796.

TONDEREAU P., r972. Influence du phosphore alimentaire sur les performances des poules pondeuses. Mémoive de fin d'études, Station de Recherches Avicoles I.N.R.A. Nouzilly.

Waldroup P. W., Simpson C. F., Damron B. L., Harms R. H., ig67. The effectiveness of plant and inorganic phosphorus in supporting egg production in hens and hatchability and bone development in chick embryos. Poultry Sci., 46, 659-664.

Waldroup P. W., Maxey J. F., Luther L. W., 1974. Studies on the calcium and phosphorus requirements of caged turkeys breeder hens. Poultry Sci., 53, 886-888. 